\title{
Anesthesia considerations of magnetic resonance imaging-guided focused ultrasound thalamotomy for essential tremor: a case series
}

\author{
Martin Chapman, B.M., FRCA, FRCPC • Andrea Park, BSc • \\ Michael Schwartz, MD, MSc · Jordan Tarshis, MD, FRCPC
}

Received: 20 February 2019/Revised: 29 January 2020/Accepted: 30 January 2020/Published online: 14 April 2020

(c) Canadian Anesthesiologists' Society 2020

\begin{abstract}
Purpose Essential tremor (ET) is a common movement disorder with disability in voluntary actions such as eating and writing. First-line treatment involves pharmacological agents, although efficacy is limited by side effects. In these patients, functional neurosurgery can be considered. Magnetic resonance imaging-guided focused ultrasound (MRgFUS) thalamotomy offers a non-invasive solution for treatment. This paper examines an original cohort of ET patients undergoing MRgFUS thalamotomy and discusses the anesthetic management of these cases.
\end{abstract}

Methods We retrospectively reviewed the anesthetic records of all MRgFUS thalamotomy cases from 15 May 2012 to 16 July 2015 at our centre (Sunnybrook Health Sciences Centre, Toronto, Canada) to expand a data set provided by the focused ultrasound system manufacturer (Insightec, Tirat Carmel, Israel) from a prior phase-II regulatory approval study. Specific drug and procedural details were listed including aspects of the patients' experience.

Results A total of 82 patients were included in the analysis, 78 from a phase-II trial (16 were from the local site) and four local non-trial cases. No patient required general anesthesia and only $29 \%$ of cases required sedation to tolerate the procedure. The most frequent medications required were antiemetics and analgesics. Headache $(31 \%)$ was the most frequent perioperative symptom. Transient intra-procedural paresthesia symptoms were a common occurrence (32\%).

Conclusions The use of MRgFUS for thalamotomy provides a non-invasive and well-tolerated method for

M. Chapman, B.M., FRCA, FRCPC ( $₫)$ · A. Park, BSc . M. Schwartz, MD, MSc · J. Tarshis, MD, FRCPC

Sunnybrook Health Sciences Centre, University of Toronto, 2075 Bayview Avenue, Toronto, ON M4N 3M5, Canada e-mail: martin.chapman@sunnybrook.ca treating ET, which usually only requires monitored anesthesia care sedation. Nevertheless, there are several predictable side effects that require contingency planning including the personnel and means to resolve them.

\section{Résumé}

Objectif Le tremblement essentiel (TE) est un trouble moteur courant qui affecte les mouvements volontaires tels que se nourrir ou écrire. Le traitement de première ligne est basé sur des agents pharmacologiques, mais son efficacité est limitée par les effets secondaires. Chez de tels patients, une neurochirurgie fonctionnelle peut être envisagée. La thalamotomie par ultrasons focalisés guidés par imagerie de résonance magnétique (ou MRgFUS) offre une solution de traitement non invasive. Cet article examine une cohorte initiale de patients souffrant de TE et subissant une thalamotomie par MRgFUS et discute de la prise en charge anesthésique de ces cas.

Méthode Nous avons évalué de manière rétrospective les dossiers anesthésiques de toutes les interventions de thalamotomie par MRgFUS réalisées entre le 15 mai 2012 et le 16 juillet 2015 dans notre centre (Sunnybrook Health Sciences Centre, Toronto, Canada) afin d'élargir un ensemble de données fourni par le fabricant de systèmes d'ultrasons focalisés (Insightec, Tirat Carmel, Israèl) basé sur une étude d'approbation règlementaire de phase II. Les médicaments spécifiques et détails procéduraux ont été enregistrés, y compris divers aspects de l'expérience des patients.

Résultats Un total de 82 patients ont été inclus dans l'analyse, dont 78 participants de l'étude de phase II (16 au site local) et quatre cas locaux non inclus dans l'étude. Aucun patient n'a nécessité d'anesthésie générale et seulement $29 \%$ des cas ont nécessité une sédation pour tolérer l'intervention. Les médicaments les plus fréquemment requis étaient les antiémétiques et 
analgésiques. Les céphalées (31 \%) constituaient le symptôme périopératoire le plus fréquent. Des symptômes de paresthésie intra-procédurale transitoire étaient fréquents (32\%).

Conclusion L'utilisation de la MRgFUS pour la thalamotomie offre une méthode non invasive et bien tolérée pour le traitement du tremblement essentiel, qui ne nécessite habituellement qu'une sédation sous surveillance comme soin anesthésique. Toutefois, cette intervention a plusieurs effets secondaires prévisibles qui nécessitent un plan de contingence incluant le personnel et les moyens pour les traiter.

Essential tremor (ET) is a common movement disorder, affecting approximately $1 \%$ of adults worldwide. ${ }^{1}$ It is generally regarded as a monosymptomatic condition that usually manifests with rest- and movement-associated hand tremor, but may also include the head, neck, and voice. The tremor frequency is typically between 7 and $12 \mathrm{~Hz} .^{2,3}$ The disability associated with ET includes impairment of voluntary actions such as drinking, eating, and writing. Treatment often involves pharmacological agents, including beta-blockers (usually propranolol) and the anti-epileptic primidone (with phenobarbital as a metabolite). The efficacy of these drugs can be limited by their side effects. Despite receiving the recommended doses of the ET medications, as many as $50 \%$ of patients develop intolerance to the medications and continue to suffer from tremor. ${ }^{4}$

In those patients whose ET symptoms are not adequately controlled pharmacologically, functional neurosurgery can be considered. Radiofrequency thalamotomy and deep brain stimulation are common neurosurgical procedures with high success rates but are invasive by nature. The Gamma knife is a non-invasive neurosurgical procedure, but has the following disadvantages: the lesion cannot be localized in real-time, it has a delayed onset, and it uses ionizing radiation for a benign condition. With the recent development of non-invasive magnetic resonance imaging (MRI)-guided focused ultrasound (MRgFUS) thalamotomy, it has become easier and safer to treat several functional neurologic disorders, including ET, with increased precision and real-time imaging. ${ }^{5,6}$

The equipment involves a helmet-mounted hemispherical phased-array series of ultrasound transducers (Fig. 1). The multiple transducers allow the acoustic energy to distribute across the skull surface, thus addressing the issue of poor bone penetration, while allowing for precise intracranial targeting. To facilitate energy transmission from the transducers to the target, all

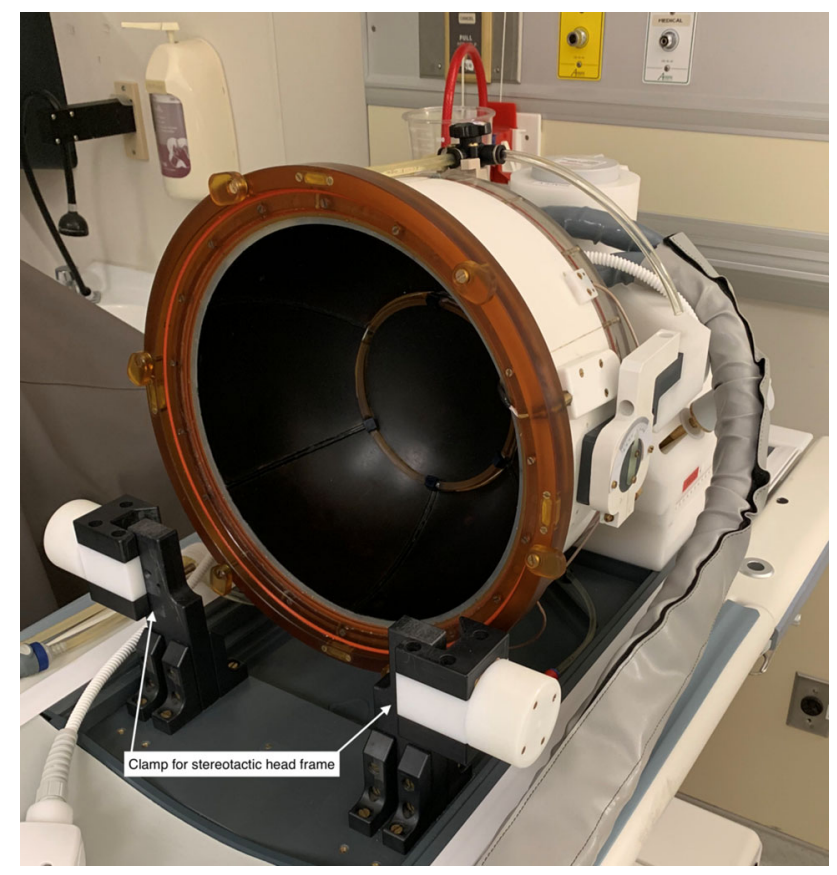

Fig. 1 Inside view of the helmet housing the ultrasound array

hair is shaved, and de-gassed cool water is circulated between the helmet-mounted transducers and the scalp. High-resolution MRI (3 T) permits real-time target temperature mapping and allows for precise intracerebral focusing of treatment location and energy deposition (Fig. 2).

The target for ablation is the ventral intermediate nucleus of the thalamus, a relay in the tremor pathways between the cerebellum and motor centres. Initially, low power sonications with a target temperature of $40-45{ }^{\circ} \mathrm{C}$ are performed to confirm targeting, followed by increased energy sonications to temperatures of $52-60{ }^{\circ} \mathrm{C}$ to achieve ablation. A phase-II randomized-controlled trial showed a $47 \%$ reduction of tremor scores at three months, with persistent symptom improvement at one year, including improvements in quality of life indicators. ${ }^{7}$

Anesthetic management for these procedures has unique challenges. Sinai et al. have reviewed some considerations, but their recommendation to avoid anxiolytics and sedatives would not be acceptable for many patients. ${ }^{8}$ This retrospective study describes the specifics of anesthetic management for this procedure.

\section{Methods}

Approval was given by our local Research Ethics Board on 6 July 2016, which granted a waiver of consent, and the data acquired were handled in compliance with local personal health information legislation. We retrospectively 
Fig. 2 Patient prior to being positioned in the magnet bore. Note that the patient's head is secured in stereotactic frame

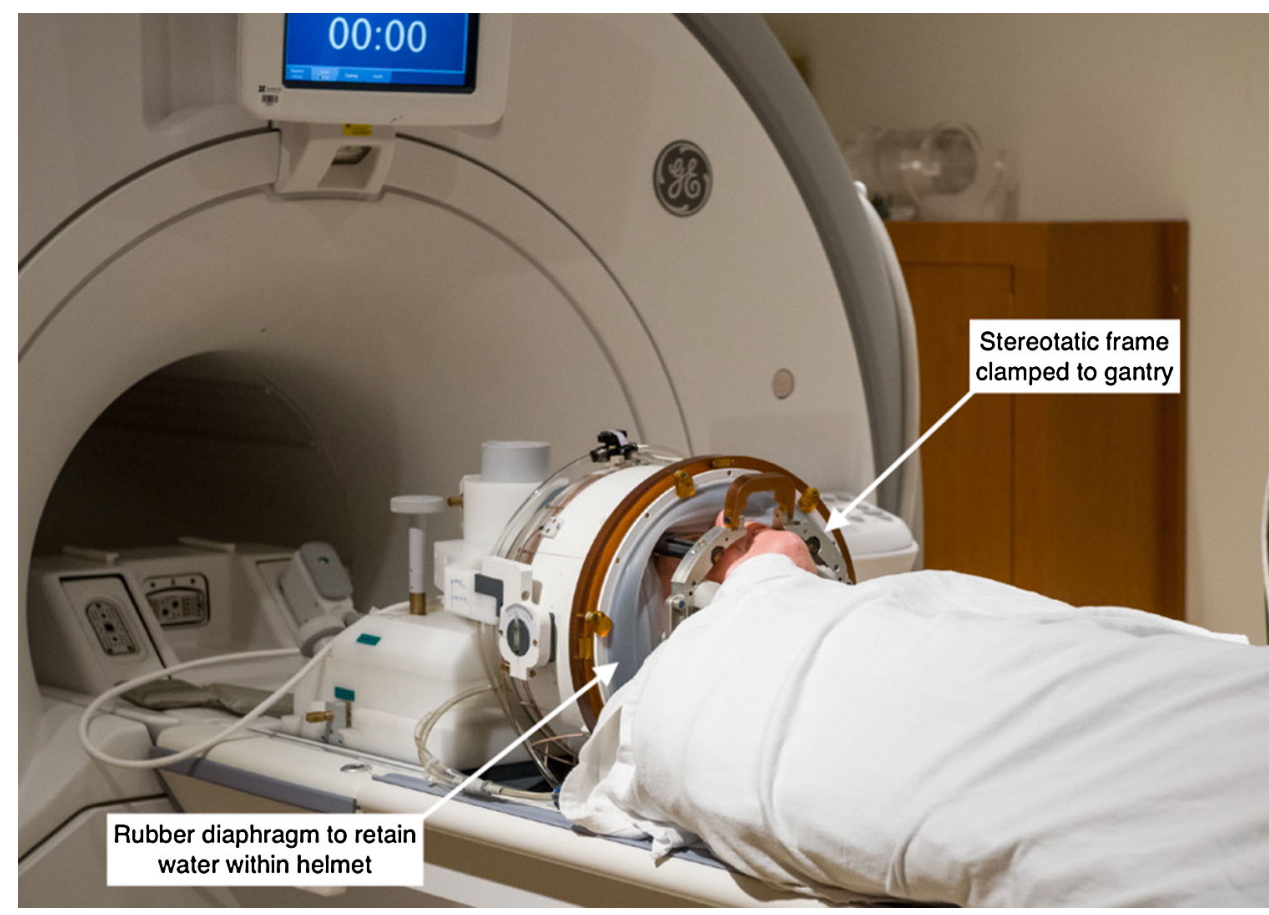

reviewed all thalamotomy cases using MRgFUS from 15 May 2012 to 16 July 2015 at our centre (Sunnybrook Health Sciences Centre, Toronto, ON, Canada). These cases were all from a Sunnybrook program of focused ultrasound (FUS) research and included locally-enrolled cases in the original phase-II regulatory approval study for MRgFUS thalamotomy in ET (ClinicalTrials.gov NCT01827904). ${ }^{7}$ These cases were identified from anesthesia billing submissions and research logs. Information was retrieved from the anesthesia records, clinical charts, and research program database, and then recorded directly to a digital clinical report form. We also accessed a larger data set from other sites in the same phase-II study collected between August 2013 and September $2014 .^{7}$ These patient data were provided by the FUS system manufacturer (Insightec, Tirat Carmel, Israel). Only patients who actually received FUS treatments were included.

Patient selection

The inclusion and exclusion criteria for all thalamotomy cases adhered to the original phase-II study protocol. ${ }^{7}$ Inclusion criteria included age $>22 \mathrm{yr}$, a diagnosis of ET as confirmed from clinical history and examination, and a postural or intention tremor severity score of 2 or more in the dominant hand or arm as measured by the clinical rating scale for tremor ${ }^{9}$ that was refractory to at least two medications. The patients were screened to exclude contraindications to MRI or neurosurgical procedures and more than mild systemic comorbidity.

Anesthetic management

Given that this was a novel therapy, familiarity with the procedure and the patient experience developed over time, and hence the anesthetic management was not standardized, neither within our site nor between sites. Table 1 describes the set-up and procedural details and the final patient position prior to moving into the magnet, as shown in Fig. 1. In all cases, head frame placement was performed awake with the use of local anesthesia to the pin sites.

Outcome assessments

All intraoperative symptoms and adverse events were collected prospectively as part of the phase-II trial protocol. $^{7}$ Details of the Sunnybrook anesthesia management were retrieved retrospectively from the anesthesia records. Only partial information regarding anesthesia was available from the multisite data set, the latter being generated from prespecified fields on the study case report form. No access to the raw data and clinical charts was available. Demographic data were summarized using simple descriptive statistics. 
Table 1 Set-up and requirements for magnetic resonance imaging-guided focused ultrasound treatment of essential tremor

\begin{tabular}{|c|c|}
\hline $\begin{array}{l}\text { Monitoring (MRI-compatible } \\
\text { monitor) }\end{array}$ & $\begin{array}{l}\text { - } \mathrm{ECG} \\
\text { - } \mathrm{NIBP} \\
\text { - } \mathrm{SpO}_{2} \\
\text { - Capnography via nasal prongs with } \mathrm{CO}_{2} \text { sampling line }\end{array}$ \\
\hline Commonly used drugs & $\begin{array}{l}\text { - Propofol } \\
\text { - Fentanyl } \\
\text { - Phenylephrine } \\
\text { - Ondansetron }\end{array}$ \\
\hline Equipment & $\begin{array}{l}\text { - SGA and T-piece with venturi adaptor on standby } \\
\text { - Nasal prongs with } \mathrm{CO}_{2} \text { sampling } \\
\text { - MRI-compatible syringe driver } \\
\text { - Resuscitation equipment including suction } \\
\text { - Patient warming device }\end{array}$ \\
\hline Procedure & $\begin{array}{l}\text { - Head shaved and stereotactic frame placed with local anesthesia outside of MRI room } \\
\text { - Local anesthetic mixture: } 10 \mathrm{~mL} 0.5 \% \text { Bupivacaine with epinephrine } 1: 200,000+9 \mathrm{~mL} 2 \% \\
\text { lidocaine }+1 \mathrm{~mL} 8.4 \% \text { sodium bicarb } \\
\text { - Antiemetic prophylaxis } \\
\text { - Compression stockings/pneumatic calf compression } \\
\text { - Ear plugs } \\
\text { - Nasal prongs placed prior to fitting membrane (part of helmet, forms water-tight seal around scalp) } \\
\text { - Patient asked to use washroom or urinary catheter placed } \\
\text { - Moved to MRI } \\
\text { - Head frame positioned and clamped to MRI table } \\
\text { - MRI-compatible monitoring: } \\
\text { a. SpO (on toe to keep arm free for neurologic testing) } \\
\text { b. Nasal cannula with } \mathrm{CO}_{2} \text { sampling } \\
\text { 1.[Propofol infusion set-up] optional } \\
\text { 2. Patient and lines monitored as moved into the magnet } \\
\text { 3. Emergency stop bulb given to patient and tested }\end{array}$ \\
\hline
\end{tabular}

$\mathrm{CO}_{2}=$ carbon dioxide; $\quad \mathrm{ECG}=$ electrocardiogram; $\quad \mathrm{MRI}=$ magnetic $\quad$ resonance $\quad$ imaging; $\quad \mathrm{NIBP}=$ non-invasive blood pressure; $\mathrm{SGA}=$ supraglottic airway; $\mathrm{SpO}_{2}=$ peripheral pulse oximetry

\section{Results}

Data from 82 patients were analyzed: 78 patients from the phase-II trial and an additional four local cases with identical selection criteria as the phase-II study. The patients' baseline characteristics and chronic health issues are listed in Table 2.

The median [interquartile range] procedure duration was 222 [190-285] min. There were brief opportunities to interact with the patient in the magnet between each sonication event, which allowed for brief symptom assessment. The most frequent medications used were antiemetics and analgesics (Table 3). To tolerate the procedure, sedation was only required in $29 \%$ of cases overall. Tolerance of the procedure was essential as preserved consciousness and patient participation were needed to aid targeting and to warn of potential complications, such as sensory deficits. Nevertheless, there was a noticeable local difference in the type of sedation used, with the Sunnybrook cases being given either propofol infusions or midazolam by an anesthesiologist, while the other sites used lorazepam exclusively. The latter sites, according to protocol, were required to have specific personnel monitoring the patient, but their qualifications were not specified (anesthesiologist, nurse anesthetist, or the treating physician). ${ }^{7}$

The stereotactic MR-compatible frame (CRW; Integra, Plainsboro, NJ, USA) was the standard arrangement as commonly used in other functional neurosurgical procedures. There was pin discomfort in $16 \%$ of cases, despite using local anesthesia in all cases. Although the sedation requirements were low, there was a need for parenteral analgesia in almost all patients, most commonly fentanyl, which was also related to pain during the 
Table 2 Patient baseline characteristics and comorbidities

\begin{tabular}{ll}
\hline & $(n=82)$ \\
\hline Gender & $58(71)$ \\
Male, $n(\%)$ & $71(8.1)$ \\
Age, mean (SD) yr & $25(5.0)$ \\
Body mass index, mean (SD) $\mathrm{kg} \cdot \mathrm{m}^{-2}$ & $83 \%$ \\
Side of brain treated & $17(10-25)$ \\
Left $(\%)$ & $5(28)$ \\
Time since diagnosis, median $[\mathrm{IQR}] \mathrm{yr}$ & $10(56)$ \\
Comorbidities, ${ }^{\mathrm{a}} n(\%)$ & $3(17)$ \\
Lung disease & $10(56)$ \\
Hypertension & \\
Diabetes mellitus & \\
Hyperlipidemia & \\
\hline IQR $=$ interquartile range; SD $=$ standard deviation & \\
a Data only available for 18 of the 20 local cases &
\end{tabular}

sonications. The most significant aspects of the patient experience are summarized in Table 3. Of note, headache $(31 \%)$, nausea $(21 \%)$, and dizziness $(14 \%)$ were the most frequent systemic symptoms. The dizziness was mostly vertiginous in nature, likely related to cerebellar projections to the target region. ${ }^{10}$ There were two cases of vomiting during the procedure. Transient intraprocedural paresthesia symptoms, mostly in the face or hand, were a common neurologic symptom (32\%).

\section{Discussion}

In this case series, we report the peri-procedural management of MRgFUS for ET. The neurologic outcome of this technique has been reported elsewhere. ${ }^{7,11}$ The patient's experience seems to be relatively innocuous and the adverse events we noted are similar to those described previously in the neurosurgical literature. ${ }^{12}$ Over the time our centre has been performing this procedure, we have found that, although it can largely be performed with the minimum of anesthetic support, there are important events that can be anticipated and require a contingency plan.

These cases had all the usual challenges of working in an MRI suite. This is related both to being in a remote site $^{13}$ and to the restrictions caused by magnetic fields. ${ }^{14}$ There are widely acknowledged standards for providing safe anesthesia in non-operating room locations ${ }^{15}$ that set out the minimum requisites for equipment and personnel. The same is also true of anesthesia in the MRI suite environment. ${ }^{16}$
With respect to MRgFUS, the compromised line of sight into the magnet necessitates excellent communication with the patient, as well as full MRI-compatible monitoring. In addition to the usual audio access into the magnet, the presence of an emergency stop button in the patient's hand seems essential when dealing with side effects that can develop over the short (seconds) treatment period. Another aspect of MRgFUS for ET that differentiates it from other MRI procedures is the comparatively longer periods, with targeting scans, to allow degassing of the helmet water, intra-procedural patient examinations, and post-sonication T2 imaging.

Part of the safety margin of the thalamotomy procedure is real-time patient feedback on symptoms (parasthesias, weakness) and how the FUS treatments are impacting the ET. With respect to the anesthetic technique, there needs to be a balance between ensuring comfort and tolerance while not masking the symptoms of ET. Beyond what we report here, there is little data available regarding the optimum mode of anesthesia. One meta-analysis of studies that compared local vs general anesthesia during subthalamic nucleus deep brain stimulation for Parkinson's disease failed to provide good-quality evidence of equivalence or superiority pertaining to patient outcomes. ${ }^{17}$ Therefore, use of sedatives is likely to be guided by institutional preferences. For example, while Sinai et al. ${ }^{8}$ refrained from using any anxiolytics to avoid reducing the tremor, the present study revealed varied use of benzodiazepines and propofol between sites. None of the sites used dexmedetomidine, which has gained some popularity for minimally invasive neurosurgery. ${ }^{18}$ Similar to other aspects of the anesthesia, there is little data to recommend the use of dexmedetomidine, with one 
Table 3 Procedural details for anesthesia in magnetic resonance imaging-guided focused ultrasound for essential tremor

\begin{tabular}{|c|c|}
\hline & $(\mathrm{n}=82)$ \\
\hline Duration of procedure, min, median [interquartile range] & $222[190-285]^{\mathrm{a}}$ \\
\hline Sedation, $n(\%)$ & $24(29)$ \\
\hline \multicolumn{2}{|l|}{ Drugs used } \\
\hline Propofol & $25 \%^{\mathrm{b}}$ \\
\hline Midazolam & $42 \%$ \\
\hline Lorazepam & $46 \%$ \\
\hline Intravenous analgesia, $n(\%)$ & $29(35)$ \\
\hline \multicolumn{2}{|l|}{ Drugs used } \\
\hline Fentanyl & $97 \%$ \\
\hline Remifentanyl & $3 \%$ \\
\hline Pentazocine & $3 \%$ \\
\hline Hydromorphone & $3 \%$ \\
\hline Oral analgesia $n(\%)$ & $58(71)$ \\
\hline \multicolumn{2}{|l|}{ Drugs used } \\
\hline Codeine/acetaminophen & $7 \%$ \\
\hline Oxycodone/acetaminophen & $4 \%$ \\
\hline Acetaminophen & $60 \%$ \\
\hline Non-steroidal anti-inflammatory drugs & $35 \%$ \\
\hline Antiemetic, $n(\%)$ & $50(61)$ \\
\hline Antacid, $n(\%)$ & $18(22)$ \\
\hline Vasodilators, $n(\%)^{\mathrm{c}}$ & $18(22)$ \\
\hline Vasopressors, $n(\%)$ & $3(4)$ \\
\hline \multicolumn{2}{|l|}{ Patient symptoms $n(\%)$ : } \\
\hline Numbness/tingling & $26(32)$ \\
\hline Headache - overall & $25(31)$ \\
\hline - sonication-related & $7(11)$ \\
\hline Nausea & $17(21)$ \\
\hline Vomiting & $2(4)^{\mathrm{d}}$ \\
\hline Pin-site pain & $10(16)^{\mathrm{e}}$ \\
\hline Dizziness & $11(14)$ \\
\hline Hypertension & $11(14)$ \\
\hline
\end{tabular}

${ }^{a}$ Only from Sunnybrook data $(n=20)$

b Five out of six patients received propofol infusions. All propofol cases from Sunnybrook site only

c No vasodilator use at Sunnybrook site

${ }^{\mathrm{d}}$ Data only from multisite data set $(n=56)$

${ }^{\mathrm{e}}$ Data only from multisite data set $(n=62)$

retrospective study showing loss of motor examination during Parkinson's disease surgery. ${ }^{19}$

There are side effects of the procedure that need to be anticipated. The most significant challenges faced were pain and the duration of the procedure. Pain occurs near the end of the treatment, when higher sonication power is being used and the patient may feel extreme heat on the scalp so that the treatment has to be aborted before completion, as occurred in five out of $56(11 \%)$ treatments in the original trial. ${ }^{7}$ One patient factor influencing the required sonication power is skull density, which may alter the amount of energy required to produce a lesion in the target area. $^{20}$ This may affect the degree of pain experienced.

The requirement for deep sedation and analgesia incurs known risks, which include aspiration. Seventeen (21\%) patients experienced nausea, which has led our site to routinely use antiemetic prophylaxis. Actual vomiting occurred in two cases in the multisite data and in three out of 15 cases $(20 \%)$ in a pilot study. ${ }^{4}$ This could 
Table 4 Anticipated side effects of the magnetic resonance imaging-guided focused ultrasound procedure and mitigation strategies

\begin{tabular}{|c|c|}
\hline Anticipated side effects & Mitigation strategies \\
\hline Headache & May experience dural irritation and pain. Mostly transient and relieved by explanation and/or opioids \\
\hline Sonication pain & $\begin{array}{l}\text { Commonly experienced at higher energies. May need intravenous analgesics, increased sedation, or may } \\
\text { need to abort procedure }\end{array}$ \\
\hline Claustrophobia & Enquire specifically pre-procedure. Maintain verbal contact via intercom. May require anxiolyics \\
\hline Vertigo \pm nausea & Antiemetics \pm sedation \\
\hline $\begin{array}{l}\text { Discomfort from prolonged } \\
\text { immobility }\end{array}$ & Low-dose propofol infusion \\
\hline Full bladder & Limit intravenous fluids, may use urinal, or insert urinary catheter \\
\hline Hypothermia & $\begin{array}{l}\text { Patient's scalp is bathed in cold water, combined with the lower temperature in the MRI suite, leads to } \\
\text { heat loss. Warm blankets and MRI-compatible warming pads (with circulating warm water) }\end{array}$ \\
\hline $\begin{array}{l}\text { Over-sedation with cardiorespiratory } \\
\text { compromise }\end{array}$ & Capnography via sampling port on nasal prongs \\
\hline
\end{tabular}

MRI = magnetic resonance imaging

compromise the airway as there is no physical way for immediate relief, due to the rigid fixation of the patient's position. It takes several minutes to release a patient from the fixed ultrasound frame. In patients who are fully conscious, this likely could be contained with adequate suctioning, albeit an unpleasant situation for the patient. Nevertheless, the risk of aspiration increases with the use of sedation. The published literature does not comment on adverse events due to nausea or vomiting.

As with any MRI procedure, maintaining an airway can be problematic. The frame and helmet create a difficult airway scenario if emergency airway control is required. ${ }^{21}$ The use of sedatives and the relatively fixed abnormal head and neck position may precipitate a degree of airway compromise; hence the need for expiratory $\mathrm{CO}_{2}$ monitoring, as per Canadian anesthesia guidelines. ${ }^{22}$ There were no cases of lost airway nor aspiration in this series. It is for these reasons that we believe that there should be a consideration given to maintaining an anesthesia care team presence throughout the procedure to optimize patient comfort and safety.

In $18(22 \%)$ cases, vasodilator agents were required to treat hypertension. It has been suggested that hypertension is avoided during treatments because of a theoretical risk of hemorrhage and may be best achieved using direct vasodilators rather than beta-blockers that may mask ET symptoms. ${ }^{23}$ That said, there is no specific evidence recommending a best practice for blood pressure control; this was reflected by differences in vasoactive drug use between the local and multisite data. ${ }^{24}$

Finally, these patients may have comorbidities in addition to ET, as patients presenting for this procedure are generally older with an American Society of Anesthesiologists (ASA) physical status $\geq$ III. Because of exclusion criteria for this novel treatment, we did not include patients with severely limiting comorbidities. As this technique becomes more widely available, it is highly probably that some ASA physical status IV patients will present for treatment.

This study is limited by the relatively small number of patients undergoing the procedure, thereby limiting some of the conclusions that can be made regarding safety and best practice. Several recent case series of Parkinson's and other ET disorders have shown a low incidence of significant adverse events during the procedure. ${ }^{12,25}$ Further experience with this technique is needed to validate these findings. Various strategies to mitigate adverse events have been included (Table 4), but these are entirely based on our own centre's experience to date.

\section{Conclusions}

Magnetic resonance imaging-guided focused ultrasound provides a non-invasive and relatively safe method for treatment of ET that is well tolerated. There are, however, several predictable side effects shown by this case series that require contingency planning and the personnel and means to resolve them.

Acknowledgements Thanks to all the members of the FUS team including Ruby Endre, Yuexi Huang, and Kullervo Hynynen, and to Mor Dayan at InSightec.

Author contributions Andrea Park, Jordan Tarshis, and Martin Chapman contributed to conception/design. Andrea Park and Martin Chapman contributed to data acquisition. All authors contributed to data analysis or data interpretation and drafting the manuscript. Jordan Tarshis and Martin Chapman contributed to manuscript correction. All authors agreed to be accountable for all aspects of the work, thereby ensuring that questions related to the accuracy or 
integrity of any part of the work will be appropriately investigated and resolved.

Funding statement This work was not supported by any source of funding.

Conflict of interest The patient data in this work originated from a study funded by InSightec, who developed the focused ultrasound technology. InSightec provided the data, reviewed the paper prior to submission, and had no other role. Andrea Park has no interests to declare. Jordan Tarshis has received research funding from BioSyent. Michael Schwartz was the principal investigator on the original study funded by Insightec, but received no personal financial support. Martin Chapman participated in the original study funded by Insightec and provided anesthesia input, but received no personal financial support.

Editorial responsibility This submission was handled by Dr. Hilary P. Grocott, Editor-in-Chief, Canadian Journal of Anesthesia.

\section{References}

1. Haubenberger D, Hallett M. Essential tremor. N Engl J Med. 2018;378:1802-10.

2. Elias WJ, Shah BB. Tremor JAMA. 2014;311:948-54.

3. Puschmann A, Wszolek ZK. Diagnosis and treatment of common forms of tremor. Semin Neurol. 2011;31:65-77.

4. Elias WJ, Huss D, Voss T, et al. A pilot study of focused ultrasound thalamotomy for essential tremor. N Engl J Med. 2013;369:640-8

5. Piper RJ, Hughes MA, Moran CM, Kandasamy J. Focused ultrasound as a non-invasive intervention for neurological disease: a review. Br J Neurosurg. 2016;30:286-93.

6. Miller DB, O'Callaghan JP. New horizons for focused ultrasound (FUS) - therapeutic applications in neurodegenerative diseases. Metabolism. 2017;69S:S3-7.

7. Elias WJ, Lipsman $N$, Ondo $W G$, et al. A randomized trial of focused ultrasound thalamotomy for essential tremor. N Engl J Med. 2016;375:730-9.

8. Sinai A, Katz Y, Zaaroor M, Sandler O, Schlesinger I. The role of the anesthesiologist during magnetic resonance-guided focused ultrasound thalamotomy for tremor: a single-center experience. Parkinsons Dis. 2018. DOI: https://doi.org/10.1155/2018/ 9764807.

9. Stacy MA, Elble RJ, Ondo WG, Wu SC, Hulihan J; TRS Study Group. Assessment of interrater and intrarater reliability of the Fahn-Tolosa-Marin tremor rating scale in essential tremor. Mov Disord 2007; 22: 833-8.

10. Wintermark $M$, Huss DS, Shah BB, et al. Thalamic connectivity in patients with essential tremor treated with MR imaging-guided focused ultrasound: in vivo fiber tracking by using diffusiontensor MR imaging. Radiology. 2014;272:202-9.

11. Lipsman N, Schwartz ML, Huang Y, et al. MR-guided focused ultrasound thalamotomy for essential tremor: a proof-of-concept study. Lancet Neurol. 2013;12:462-8.
12. Zaaroor M, Sinai A, Goldsher D, Eran A, Nassar M, Schlesinger I. Magnetic resonance-guided focused ultrasound thalamotomy for tremor: a report of 30 Parkinson's disease and essential tremor cases. J Neurosurg. 2018;128:202-10.

13. Melloni $C$. Morbidity and mortality related to anesthesia outside the operating room. Minerva Anestesiol. 2005;71:325-34.

14. Berkow LC. Anesthetic management and human factors in the intraoperative MRI environment. Curr Opin Anaesthesiol. 2016;29:563-7.

15. American Society of Anesthesiologists. Statement on Nonoperating Room Anesthetizing Locations. Committee of Origin: Standards and Practice Parameters. (Approved by the ASA House of Delegates on October 19, 1994, last amended on October 16, 2013, and reaffirmed on October 17, 2018). Available from URL: https://www.asahq.org/standards-andguidelines/statement-on-nonoperating-room-anesthetizinglocations. Accessed Jan 2020.

16. American Society of Anesthesiologists Task Force. Practice advisory on anesthetic care for magnetic resonance imaging: an updated report on Anesthetic Care for Magnetic Resonance Imaging. Anesthesiology 2015;122:495-520.

17. Sheshadri V, Rowland NC, Mehta J, Englesakis M, Manninen P, Venkatraghavan L. Comparison of general and local anesthesia for deep brain stimulator insertion: a systematic review. Can J Neurol Sci. 2017;44:697-704.

18. Suero Molina E, Schipmann S, Mueller I, et al. Conscious sedation with dexmedetomidine compared with asleep-awakeasleep craniotomies in glioma surgery: an analysis of 180 patients. J Neurosurg. 2018;129:1223-30.

19. Honorato-Cia C, Martínez-Simón A, Alegre M, et al. Factors associated with tremor changes during sedation with dexmedetomidine in Parkinson's disease surgery. Stereotact Funct Neurosurg. 2015;93:393-9.

20. Schwartz ML, Yeung R, Huang Y, et al. Skull bone marrow injury caused by MR-guided focused ultrasound for cerebral functional procedures. J Neurosurg. 2018;130:758-62.

21. York JE, Wharen RE, Bloomfield EL. Esophageal tear in a patient undergoing stereotactic brain biopsy under general anesthesia. J Anesth. 2009;23:432-5.

22. Dobson G, Chow L, Flexman A, et al. Guidelines to the practice of anesthesia - revised edition 2019. Can J Anesth 2019; 66: 75108.

23. Dunn LK, Durieux ME, Elias WJ, Nemergut EC, Naik BI. Innovations in functional neurosurgery and anesthetic implications. J Neurosurg Anesthesiol. 2018;30:18-25.

24. McDannold N, Zhang Y, Vykhodtseva N. Blood-brain barrier disruption and vascular damage induced by ultrasound bursts combined with microbubbles can be influenced by choice of anesthesia protocol. Ultrasound Med Biol. 2011;37:1259-70.

25. Sinai A, Nassar M, Eran A, et al. Magnetic resonance-guided focused ultrasound thalamotomy for essential tremor: a 5-year single-center experience. J Neurosurg. 2019. DOI: https://doi.org/ 10.3171/2019.3.JNS19466.

Publisher's Note Springer Nature remains neutral with regard to jurisdictional claims in published maps and institutional affiliations. 